\title{
Approximate linear minimum mean square error estimation based on channel quality indicator feedback in LTE systems
}

\begin{abstract}
The fast fading channel produced by the fast user mobility requires a powerful channel estimation to report the most accurate channel status. Such estimation techniques are usualy suffer from large number of computational processes, and thus, their complexity needs to be minimized. However, the calculation reliability of channel coefficients depends mainly on the accuracy of the channel estimation model. Therefore, obtaining a joint optimized solution for channel estimation error, feedback overhead, and complexity is very crucial. In this paper, two different channel estimation schemes; Linear Minimum Mean Square Error (LMMSE) and Approximate Linear Minimum Mean Square Error (ALMMSE) are used to calculate Channel Quality Indicator (CQI) and Precoding Matrix Indicator (PMI) in the 3GPP-LTE fast fading channel. It is found that, by using a low-complexity ALMMSE, the estimation error is reduced with relatively small reduction in throughput. Therefore, the proposed method is recommended to be used when the network is not fully loaded for better tradeoff concerning MSE and throughput taking into account the fixed and mobility scenarios, and thus, reliable transmission will be targeted.
\end{abstract}

Keyword: 3GPP LTE/ LTE-A; OFDM; Channel quality indicator (CQI); Precoding matrix indicator PMI; Channel estimation; ALMMSE 